\title{
ON EXTENDED EULERIAN NUMBERS
}

\author{
Abdelmejid Bayad, Mohand Ouamar Hernane, Alain Togbé
}

\begin{abstract}
In this paper, we will study some properties of the extended Eulerian numbers $H(n, \lambda)$, with a parameter $\lambda$. In fact, for any integer $n$, we investigate the asymptotic behavior, find lower and upper bounds for $H(n, \lambda)$. As application, for a champion number $N$, we obtain asymptotic formulas, lower and upper bounds of the arithmetic functions $\omega(N)$ and $\Omega(N)$.
\end{abstract}

Keywords: Kalmar's function, extended Eulerian numbers, Champion numbers, asymptotic formula, Ikehara-Wiener theorem.

\section{Introduction and preliminaries}

Let $\lambda$ be a complex number. An extended Eulerian number $H(n, \lambda)$ is defined by means of its Dirichlet series

$$
\frac{\lambda-1}{\lambda-\zeta(s)}=\sum_{n \geqslant 1} \frac{H(n, \lambda)}{n^{s}},
$$

where $\zeta(s)=\sum_{n \geqslant 1} \frac{1}{n^{s}}$ is the Riemann Zeta function defined for $\Re(s)>1$. A champion number $N$ for the function $H$ is a number that satisfies

$$
n<N \Longrightarrow H(n, \lambda)<H(N, \lambda)
$$

In this paper, we will study several properties of extended Eulerian numbers $H(n, \lambda)$, with a real parameter $\lambda>1$. We will extend and improve some results obtained by Kalmár [17], Hille [14], Erdös [8], Evans [9], Klazar and Luca [18], Deleglise, Hernane, and Nicolas [13], concerning the maximal order of extended Eulerian numbers. As an application of our results, we investigate the $H$-champion numbers.

We are motivated by the following important particular cases:

1) If $\lambda=0$, then $H(n, 0)=\mu(n)$ is a Möbius number.

2010 Mathematics Subject Classification: primary: 11A25; secondary: 11N37, 49K10 
2) If $n=p_{1} p_{2} \cdots p_{r}$ is square-free, then $H(n, \lambda)=H_{r}(\lambda)$, where $H_{r}(\lambda)$ is an Eulerian number. In addition, if $\lambda=-1$, thus

$$
H(n,-1)=H_{r}(-1)=E_{r}
$$

is the so-called Euler number. One can notice that an Euler number $E_{n}$ corresponds to $2^{-n} C_{n}$ in [21, p.28]. One can also see [3, p.688] and [1, p.354, formula (1.1.5)].

3) If $\lambda=2$, then $H(n, 2)=K(n)$, where $K$ is the Kalmár arithmetic function which counts the number of ordered factorizations of a positive integer $n$ in factors bigger than 1. Various properties of this function were studied by many mathematicians. In fact, Kalmár found the average order of $K(n)$, for $x \rightarrow \infty$

$$
\sum_{n \leqslant x} K(n)=-\frac{x^{\rho}}{\rho \zeta^{\prime}(\rho)}\{1+o(1)\}
$$

where $\rho=1.72864 \ldots$ is the positive real solution to $\zeta(s)=2$. On the other hand, this result was improved by Hwang [15]. Moreover, bounds on the maximal order of $K(n)$ were studied by Erdös [8]), Chor, Lemke and Mador [5], Coppersmith and Lewenstein [6], and Hille [14]. Recently, Klazar and Luca [18], Deleglise, Hernane, and Nicolas [13] improved the bounds for the maximal order of $K(n)$.

It is well-known that the extended Eulerian numbers $H(n, \lambda)$ satisfy the following properties:

1) The recurrence formulas are given by

$$
\left\{\begin{array}{l}
\lambda H(n, \lambda)=\sum_{d \mid n} H(d, \lambda), \quad n \geqslant 2, \lambda \neq 1, \\
H(1, \lambda)=1
\end{array}\right.
$$

See formula (1.15) in [3].

2) The expression $(\lambda-1)^{\Omega(n)} H(n, \lambda)$ is a polynomial in $\lambda$ of degree less than $\Omega(n)$, where $\Omega(n)=\sum_{k=1}^{r} k_{i}$, if $n=p_{1}^{k_{1}} p_{2}^{k_{2}} \cdots p_{r}^{k_{r}}$. In the special case where $n=p_{1} p_{2} \cdots p_{r}$, we have

$$
(\lambda-1)^{\Omega(n)} H(n, \lambda)=(\lambda-1)^{r} H_{r}(\lambda)=\sum_{t=1}^{r} A_{r, t} \lambda^{t-1}, \quad r \geqslant 1
$$

with $A_{r, t}=\sum_{j=0}^{t}(-1)^{j}\left(\begin{array}{c}r+1 \\ j\end{array}\right)(t-j)^{r}$. See formulas (5.12), (5.13) in [3].

Writing

$$
\frac{\lambda-1}{\lambda-\zeta(s)}=\frac{\lambda-1}{\lambda} \frac{1}{1-\frac{\zeta(s)}{\lambda}}
$$

and expanding it, we obtain the explicit formula

$$
H(n, \lambda)=\frac{\lambda-1}{\lambda} \sum_{k=1}^{\Omega(n)} \frac{d_{k}(n)}{\lambda^{k}}, \quad \text { where } d_{k}(n)=\sum_{\substack{n_{1} n_{2} \cdots n_{k}=n \\ n_{1}, n_{2}, \ldots, n_{k} \geqslant 1}} 1 .
$$


If we rewrite $\frac{\lambda-1}{\lambda-\zeta(s)}=\frac{1}{1-\left(\frac{\zeta(s)-1}{\lambda-1}\right)}$ and expand it, we obtain another explicit formula proved in [10]

$$
H(n, \lambda)=\sum_{k=1}^{\Omega(n)} \frac{d_{k}^{\prime}(n)}{(\lambda-1)^{k}}, \quad \text { where } d_{k}^{\prime}(n)=\sum_{\substack{n_{1} n_{2} \cdots n_{k}=n \\ n_{1}, n_{2}, \ldots, n_{k} \geqslant 2}} 1 .
$$

For $\lambda>1$, we consider the Mellin inverse integral

$$
\frac{1}{2 \pi i} \int_{\sigma-i \infty}^{\sigma+i \infty} \frac{\lambda-1}{\lambda-\zeta(s)} x^{s} \frac{d s}{s}=\sum_{n \geqslant 1} H(n, \lambda) \int_{\sigma-i \infty}^{\sigma+i \infty}\left(\frac{x}{n}\right)^{s} \frac{d s}{s}
$$

by Perron's formula, we get

$$
\frac{1}{2 \pi i} \int_{\sigma-i \infty}^{\sigma+i \infty} \frac{\lambda-1}{\lambda-\zeta(s)} x^{s} \frac{d s}{s}=\sum_{n \leqslant x}^{\star} H(n, \lambda) .
$$

Here, the symbol * on the summation indicates that the last term of the sum must be multiplied by $1 / 2$ when $x$ is an integer. Now, using Ikehara-Wiener theorem, we obtain the average formula for extended Eulerian numbers

$$
\sum_{n \leqslant x}^{\star} H(n, \lambda) \sim C(\lambda) x^{\rho(\lambda)}
$$

where $\rho(\lambda)$ is the positive real number solution to the equation $\zeta(s))=\lambda$ and

$$
C(\lambda)=\frac{1-\lambda}{\rho(\lambda) \zeta^{\prime}(\rho(\lambda))} .
$$

Formula (1.5) was proved by Evans [9]. Formulas (1.6), (1.8) and (1.9) were also obtained by Grosswald [10].

In this paper, we will study the behavior of the functions $\lambda \mapsto C(\lambda)$ and $\lambda \mapsto \rho(\lambda)$. As an application of this study, we will investigate $H$-champions numbers, specially their asymptotic, lower, and upper bounds. The size of the exponents of their prime factors will be estimated.

\section{Statement of the main results}

Let $k$ be a positive integer, $\mathcal{N}_{k}$ a multiplicative system (including 1) associated to the set of primes numbers, $\mathcal{P}_{k}=\left\{p_{1}, p_{2}, \ldots, p_{k}\right\}$, with $p_{1}=2<p_{2}=3<\cdots<p_{k}$. Define

$$
\zeta_{k}(s)=\prod_{p \in \mathcal{P}_{k}}\left(1-p^{-s}\right)^{-1},
$$

which is equivalent to

$$
\zeta_{k}(s)=\sum_{n \in \mathcal{N}_{k}} \frac{1}{n^{s}}
$$


Let $\rho_{k}(\lambda)$ be the positive real solution to $\zeta_{k}(s)=\lambda$ and $a_{k}(\lambda)=-\frac{\lambda}{\zeta_{k}^{\prime}\left(\rho_{k}(\lambda)\right)}$. For $k=\infty$, we get the Riemann zeta function $\zeta(s)$ and $\rho(\lambda)=\rho_{\infty}(\lambda), \zeta(\rho(\lambda))=\lambda$ and $a(\lambda)=-\frac{\lambda}{\zeta^{\prime}(\rho(\lambda))}$. We state our first main result.

Theorem 1. Let $\lambda>1$. Then

1. The function $\lambda \mapsto \rho(\lambda)$ is strictly increasing and $\rho(\lambda)>1$, for any $\lambda>1$.

2. The sequence $\left(\rho_{k}(\lambda)\right)_{k \geqslant 1}$ is strictly increasing and bounded. Moreover, for $\Re(s) \geqslant \sigma>1$, the function $\zeta_{k}(s)$ uniformly converges to $\zeta(s)$ as $k$ tends to $\infty$.

3. We have

$$
\rho(\lambda)-\rho_{k}(\lambda)=\frac{\lambda}{(\rho(\lambda)-1)\left|\zeta^{\prime}(\rho(\lambda))\right| k^{\rho(\lambda)-1}(\log k)^{\rho(\lambda)}}\left\{1+O\left(\frac{\log \log k}{\log k}\right)\right\} .
$$

4. The sequence $\left(a_{k}(\lambda)\right)$ is decreasing and converges to $a(\lambda)$, as $k$ tends to $\infty$. Moreover, there exists a positive constant $\gamma(\lambda)$, depending only on $\lambda$, such that for any prime $p$, we have the inequality

$$
\left|\frac{a_{k}(\lambda)}{p^{\rho_{k}(\lambda)}-1}-\frac{a(\lambda)}{p^{\rho(\lambda)}-1}\right| \leqslant \gamma(\lambda) \frac{\log p}{p^{\rho_{2}(\lambda)}(k \log k)^{\rho_{k}(\lambda)-1}} .
$$

To state our remaining main results, we need some notations and definitions. So let $\underline{x}=\left(x_{j}\right)_{1 \leqslant j \leqslant \omega}$ be a finite or infinite sequence of positive real numbers such that

$$
0<\Omega(\underline{x})=\sum_{j=1}^{\omega} x_{j}<\infty .
$$

There exists a unique $C=C(\underline{x})>0$ such that

$$
\prod_{j=1}^{\omega}\left(1+\frac{x_{j}}{C(\underline{x})}\right)=\lambda \text {. }
$$

Put

and

$$
\begin{aligned}
& T(\underline{x})=\sum_{i=1}^{\omega} \frac{x_{i}}{x_{i}+C(\underline{x})}, \\
& A(\underline{x})=\frac{\lambda-1}{\lambda \sqrt{\lambda}} \exp (-\Omega(\underline{x})) \prod_{i=1}^{\omega} \frac{\left(x_{i}+C(\underline{x})\right)^{x_{i}}}{\Gamma\left(x_{i}+1\right)}
\end{aligned}
$$

$$
B(\underline{x})=\sqrt{\frac{2 C(\underline{x})}{T(\underline{x})}} .
$$

If $n=q_{1}^{\alpha_{1}} q_{2}^{\alpha_{2}} \cdots q_{k}^{\alpha_{k}}, \underline{\alpha}=\left(\alpha_{1}, \alpha_{2}, \cdots, \alpha_{k}\right), \alpha_{i}>0, \Omega=\Omega(\underline{\alpha})$, R. Evans [9] proved the following important result

$$
H(n, \lambda)=\sqrt{\pi} A(\underline{\alpha}) B(\underline{\alpha})\left\{1+O\left(\Omega^{-F}\right)\right\},
$$

when $\Omega(\underline{\alpha})$ tends to $\infty$, for any $F$ such that $0<F<\frac{1}{2}$. Next, we state main important inequalities needed in our study. 
Theorem 2. For $\lambda>1$, we have

$$
\begin{aligned}
\frac{\Omega(\underline{x})}{\lambda-1} & <C(\underline{x}) \leqslant \frac{\Omega(\underline{x})}{\log \lambda}, \\
\frac{\lambda-1}{\lambda} & \leqslant T(\underline{x}) \leqslant \lambda-1, \\
\sqrt{\frac{2}{\lambda-1} C(\underline{x})} & \leqslant B(\underline{x}) \leqslant \sqrt{\frac{2 \lambda}{\lambda-1} C(\underline{x})} .
\end{aligned}
$$

If $n=2^{\alpha_{1}} 3^{\alpha_{2}} \cdots p_{k}^{\alpha_{k}}, \alpha_{1}, \alpha_{2}, \ldots, \alpha_{k} \geqslant 1$, then there exist absolute positive constants $C_{1}$ and $C_{2}$ such that we have

$$
C_{1} \frac{\exp (-k)}{\lambda \sqrt{\lambda}} \frac{\exp (F(\underline{\alpha}))}{\sqrt{\alpha_{1} \alpha_{2} \cdots \alpha_{k}}} \leqslant H(n, \lambda) \leqslant C_{2} \frac{1}{\lambda} \sqrt{\frac{\lambda-1}{\log \lambda}} \frac{n^{\rho_{k}(\lambda)}}{\pi^{k / 2}},
$$

where

$$
F(\underline{x})=\sum_{j=1}^{k} x_{j} \log \left(1+\frac{C(\underline{x})}{x_{j}}\right)
$$

Put

$$
f(\lambda)=C_{2} \frac{1}{\lambda} \sqrt{\frac{\lambda-1}{\log \lambda}}, C_{2}>0 \quad \text { and } \quad g(\lambda)=C_{1} \frac{1}{\lambda \sqrt{\lambda}} .
$$

Theorem 3. Let $n$ be a positive integer and $N$ an $H$-champion number.

1) For $n$ large, we have

$$
\log H(n, \lambda) \leqslant U(n, \lambda)
$$

where

$$
U(n, \lambda)=\rho(\lambda) \log n-\gamma_{1}(\lambda) \frac{(\log n)^{1 / \rho(\lambda)}}{\log \log n}
$$

and

$$
\gamma_{1}(\lambda)=\min \left(\frac{1}{2}, \frac{\lambda}{(\rho(\lambda)-1)\left|\zeta^{\prime}(\rho(\lambda))\right|}\right) .
$$

2) For a large $H$-champion number $N$, there exist a positive constant $\gamma_{2}(\lambda)$ depending only on $\lambda$ such that

$$
L(N, \lambda) \leqslant \log H(N, \lambda) \leqslant U(N, \lambda)
$$

where

$$
L(N, \lambda)=\rho(\lambda) \log N-\gamma_{2}(\lambda) \frac{(\log N)^{1 / \rho(\lambda)}}{\log \log N}+\log g(\lambda)-\rho(\lambda) \log 2 .
$$

3) For a large $H$-champion number $N$, there exist $\gamma_{3}(\lambda), \gamma_{4}(\lambda)>0$ such that

$$
\gamma_{3}(\lambda) \frac{(\log N)^{1 / \rho(\lambda)}}{\log \log N} \leqslant \omega(N) \leqslant \gamma_{4}(\lambda) \frac{(\log N)^{1 / \rho(\lambda)}}{\log \log N} .
$$


Here is the statement of our last result.

Theorem 4. Let $N$ be a H-champion number and $\delta(\lambda)=\left(1+\frac{1}{\rho(\lambda)}\right) / 2$. Then, for

$$
N=2^{\alpha_{1}} 3^{\alpha_{2}} \cdots p_{k}^{\alpha_{k}}
$$

such that $N$ tends to $\infty$, we have

1. $\Omega(N)=\sum_{i=1}^{k} \alpha_{i}=a(\lambda) \log N \sum_{i=1}^{k} \frac{1}{p_{i}^{\rho(\lambda)}-1}+O\left((\log N)^{\delta(\lambda)}\right)$ and $\alpha_{i}=$ $\frac{a(\lambda)}{p_{i}^{\rho(\lambda)}-1} \log N+O\left(\frac{(\log N)^{\delta(\lambda)}}{\log p_{i}}\right)$;

2. $C(\underline{\alpha})=a(\lambda) \log N+O\left((\log N)^{\delta(\lambda)}\right)$;

3. $T(\underline{\alpha})=\zeta_{\mathcal{P}}(\rho(\lambda))+O\left((\log N)^{\delta(\lambda)-1}\right)$;

4. $B(\underline{\alpha})=\sqrt{\frac{2 a(\lambda)}{\zeta_{\mathcal{P}}(\rho(\lambda))} \log N}\left\{1+O\left((\log N)^{\delta(\lambda)-1}\right)\right\}$;

5. $H(N, \lambda)=\sqrt{\frac{2 a(\lambda)}{\zeta_{\mathcal{P}}(\rho(\lambda))} \pi \log N} A(\underline{\alpha})\left\{1+O\left((\log N)^{\delta(\lambda)-1}\right)\right\}$.

The next sections will be devoted to the proofs of the above theorems.

\section{Proof of Theorem 1}

Let $k \geqslant 2$. There exist unique positive real numbers $\rho_{k}(\lambda), \rho(\lambda)$ such that $\zeta_{k}\left(\rho_{k}(\lambda)\right)=$ $\zeta(\rho(\lambda))=\lambda$. Then, we have

$$
\prod_{p \in \mathcal{P}_{k}}\left(1-p^{-\rho_{k}(\lambda)}\right)^{-1}=\prod_{p \in \mathcal{P}}\left(1-p^{-\rho(\lambda)}\right)^{-1}=\lambda .
$$

Thus, we obtain

$$
\lambda=\prod_{p \in \mathcal{P}_{k}}\left(1-p^{-\rho_{k}(\lambda)}\right)^{-1}=\prod_{p \in \mathcal{P}_{k}}\left(1-p^{-\rho_{k}(\lambda)}\right)^{-1} \prod_{p>p_{k}}\left(1-p^{-\rho(\lambda)}\right)^{-1} .
$$

Taking the logarithm of the above equation, we get

$$
\log \zeta_{k}\left(\rho_{k}(\lambda)\right)-\log \zeta_{k}(\rho(\lambda))=-\sum_{p>p_{k}} \log \left(1-p^{-\rho(\lambda)}\right)
$$

For fixed $\lambda>1$, there exists a positive integer $m(\lambda) \geqslant 2$ such that for all $k \geqslant m(\lambda)$ we have $\rho_{k}(\lambda)>1$. By Lagrange's mean-value, we obtain

$$
\log \zeta_{k}\left(\rho_{k}(\lambda)\right)-\log \zeta_{k}(\rho(\lambda))=\left(\rho(\lambda)-\rho_{k}(\lambda)\right) \sum_{p \in \mathcal{P}_{k}} \frac{\log p}{p^{\sigma_{p}(\lambda)}-1},
$$

for some $\sigma_{p}(\lambda) \in\left(\rho_{k}(\lambda), \rho(\lambda)\right) \subset(1,2)$. We have

$$
-\sum_{p>p_{k}} \log \left(1-p^{-\rho(\lambda)}\right)=\sum_{p>p_{k}} \frac{1}{p^{\rho(\lambda)}}+O\left(\sum_{p>p_{k}} \frac{1}{p^{2 \rho(\lambda)}}\right),
$$


and by standard estimates we get

$$
\sum_{p>p_{k}} \frac{1}{p^{\rho(\lambda)}}=\frac{(\rho(\lambda)-1)^{-1}}{p_{k}^{\rho(\lambda)-1} \log p_{k}}+O\left(\frac{1}{p_{k}^{\rho(\lambda)-1}\left(\log p_{k}\right)^{2}}\right)
$$

since $p_{k}=k \log k+k \log \log k+O(k)$. Therefore, we obtain

$$
-\sum_{p>p_{k}} \log \left(1-p^{-\rho(\lambda)}\right)=\frac{(\rho(\lambda)-1)^{-1}}{k^{\rho(\lambda)-1}(\log k)^{\rho(\lambda)}}\left\{1+O\left(\frac{\log \log k}{\log k}\right)\right\} .
$$

On the other hand, we have

$$
\frac{\log p}{p^{\sigma_{p}(\lambda)}-1}=\frac{\log p}{p^{\sigma_{p}(\lambda)}-1}\left\{1+\frac{p^{\sigma_{p}(\lambda)}}{p^{\sigma_{p}(\lambda)}-1}\left(p^{\rho(\lambda)-\sigma_{p}(\lambda)}-1\right)\right\} .
$$

Note that

$$
1 \leqslant \frac{p^{\sigma_{p}(\lambda)}}{p^{\sigma_{p}(\lambda)}-1} \leqslant 2
$$

and for $2 \leqslant p \leqslant p_{k}$, we have

$$
\begin{aligned}
p^{\rho(\lambda)-\sigma_{p}(\lambda)}-1 \leqslant p_{k}^{\rho(\lambda)-\rho_{k}(\lambda)}-1 & =\exp \left(\left(\rho(\lambda)-\rho_{k}(\lambda)\right) \log p_{k}\right)-1 \\
& \ll\left(\rho(\lambda)-\rho_{k}(\lambda)\right) \log p_{k} \\
& \ll \frac{1}{k^{\rho(\lambda)-1}(\log k)^{\rho(\lambda)-1}} .
\end{aligned}
$$

For these inequalities, we used $\exp (x) \ll x$, when $x$ tends to 0 and

$$
\rho(\lambda)-\rho_{k}(\lambda) \ll \frac{1}{k^{\rho(\lambda)-1}(\log k)^{\rho(\lambda)}},
$$

since $p_{k}=k \log k+k \log \log k+O(k)$. We remark that

$$
\begin{aligned}
\log \zeta_{k}(\rho(\lambda))- & \log \zeta_{k}\left(\rho_{k}(\lambda)\right) \\
& =\left(\rho(\lambda)-\rho_{k}(\lambda)\right) \sum_{p \in \mathcal{P}_{k}} \frac{\log p}{p^{\sigma_{p}(\lambda)}-1} \\
& =\left(\rho(\lambda)-\rho_{k}(\lambda)\right)\left(\frac{\log 2}{2^{\sigma_{p}(\lambda)}-1}+\frac{\log 3}{3^{\sigma_{p}(\lambda)}-1}+\cdots+\frac{\log p_{k}}{p_{k}^{\sigma_{p}(\lambda)}-1}\right) \\
& >\left(\rho(\lambda)-\rho_{k}(\lambda)\right) \frac{\log 2}{2^{\sigma_{p}(\lambda)}-1}, \quad \text { with } \sigma_{p}(\lambda)>1 .
\end{aligned}
$$


From (3.3), (3.8), (3.10), and (3.12), we obtain

$$
\begin{aligned}
\left(\rho(\lambda)-\rho_{k}(\lambda)\right) \sum_{p \in \mathcal{P}_{k}} \frac{\log p}{p^{\sigma_{p}(\lambda)}-1}= & \left(\rho(\lambda)-\rho_{k}(\lambda)\right) \sum_{p \in \mathcal{P}_{k}} \frac{\log p}{p^{\rho(\lambda)}-1} \\
& \times\left\{1+O\left(k^{1-\rho(\lambda)}(\log k)^{1-\rho(\lambda)}\right)\right\} \\
= & \left(\rho(\lambda)-\rho_{k}(\lambda)\right) \sum_{p \in \mathcal{P}_{k}} \frac{\log p}{p^{\rho(\lambda)}-1}\left\{1+O\left(k^{1-\rho(\lambda)}\right)\right\} .
\end{aligned}
$$

Therefore, from (3.3) and (3.7), we get

$$
\left(\rho(\lambda)-\rho_{k}(\lambda)\right) \sum_{p \in \mathcal{P}_{k}} \frac{\log p}{p^{\sigma_{p}(\lambda)}-1}=\frac{(\rho(\lambda)-1)^{-1}}{k^{\rho(\lambda)-1}(\log k)^{\rho(\lambda)}}\left\{1+O\left(\frac{\log \log k}{\log k}\right)\right\}
$$

On the other hand, we have

$$
\frac{\left|\zeta^{\prime}(\rho(\lambda))\right|}{\zeta(\rho(\lambda))}=\sum_{p \in \mathcal{P}} \frac{\log p}{p^{\rho(\lambda)}-1}=\sum_{p \in \mathcal{P}_{k}} \frac{\log p}{p^{\rho(\lambda)}-1}+\sum_{p>p_{k}} \frac{\log p}{p^{\rho(\lambda)}-1}
$$

From (3.6), we deduce

$$
\frac{\left|\zeta^{\prime}(\rho(\lambda))\right|}{\lambda}=\sum_{p \in \mathcal{P}_{k}} \frac{\log p}{p^{\rho(\lambda)}-1}+O\left(k^{1+\varepsilon-\rho(\lambda)}\right)
$$

for any small $\varepsilon>0$ and for any integer $k$ such that $k>m(\lambda)$. Thus, we get

$$
\begin{aligned}
\left(\rho(\lambda)-\rho_{k}(\lambda)\right)\left[\frac{\left|\zeta^{\prime}(\rho(\lambda))\right|}{\lambda}+\right. & \left.O\left(k^{1+\varepsilon-\rho(\lambda)}\right)\right] \\
& =\frac{(\rho(\lambda)-1)^{-1}}{k^{\rho(\lambda)-1}(\log k)^{\rho(\lambda)}}\left\{1+O\left(\frac{\log \log k}{\log k}\right)\right\}
\end{aligned}
$$

which implies that

$$
\rho(\lambda)-\rho_{k}(\lambda)=\frac{\lambda}{(\rho(\lambda)-1)\left|\zeta^{\prime}(\rho(\lambda))\right| k^{\rho(\lambda)-1}(\log k)^{\rho(\lambda)}}\left\{1+O\left(\frac{\log \log k}{\log k}\right)\right\},
$$

$\forall k>m(\lambda)$. 
Now, we will prove relation (2.4). We apply the mean-value theorem to the function $x \mapsto \frac{1}{p^{x}-1}$ to obtain

$$
\begin{aligned}
\frac{a_{k}(\lambda)}{p^{\rho_{k}(\lambda)}-1}-\frac{a(\lambda)}{p^{\rho(\lambda)}-1} & =\left(\frac{1}{p^{\rho_{k}(\lambda)}-1}-\frac{1}{p^{\rho(\lambda)}-1}\right) a_{k}(\lambda)+\frac{a_{k}(\lambda)-a(\lambda)}{p^{\rho(\lambda)}-1} \\
& =\left(\rho(\lambda)-\rho_{k}(\lambda)\right) a_{k}(\lambda) \frac{p^{x_{0}} \log p}{\left(p^{x_{0}}-1\right)^{2}}+\frac{a_{k}(\lambda)-a(\lambda)}{p^{\rho(\lambda)}-1}
\end{aligned}
$$

with $\rho_{k}(\lambda) \leqslant x_{0} \leqslant \rho(\lambda)$. Moreover, we get

$$
\begin{gathered}
\frac{p^{x_{0}}}{\left(p^{x_{0}}-1\right)^{2}} \leqslant \frac{p^{\rho_{2}(\lambda)}}{\left(p^{\rho_{2}(\lambda)}-1\right)^{2}} \leqslant \frac{C(\lambda)^{2}}{p^{\rho_{2}(\lambda)}} \\
\frac{1}{p^{x_{0}}-1} \leqslant \frac{1}{p^{\rho_{2}(\lambda)}-1} \leqslant \frac{1}{p^{\rho_{2}(\lambda)}} \cdot C(\lambda) \leqslant \frac{3 \rho_{3}(\lambda) C(\lambda) \log p}{2 \log 2} \cdot \frac{1}{p^{\rho_{2}(\lambda)}},
\end{gathered}
$$

where

$$
C(\lambda)=\frac{2^{\rho_{2}(\lambda)}}{2^{\rho_{2}(\lambda)}-1} .
$$

On the other hand, $\left(a_{k}(\lambda)\right)_{k}$ is a decreasing sequence such that

$$
\frac{2^{\rho_{2}(\lambda)}-1}{\log 3} \leqslant a_{2}(\lambda) \leqslant \frac{3^{\rho_{3}(\lambda)}}{2 \log 2} \leqslant \frac{3^{\rho_{3}(\lambda)}}{2}
$$

and

$$
\begin{aligned}
\mid \frac{a_{k}(\lambda)}{p^{\rho_{k}(\lambda)}-1} & -\frac{a(\lambda)}{p^{\rho(\lambda)}-1} \mid \\
& \leqslant \frac{3^{\rho_{3}(\lambda)} \log p}{2 p^{\rho_{2}(\lambda)}}\left[C(\lambda)^{2}\left(\rho(\lambda)-\rho_{k}(\lambda)\right)+C(\lambda)\left(a_{k}(\lambda)-a(\lambda)\right)\right] .
\end{aligned}
$$

We have

$$
a_{k}(\lambda)-a(\lambda)=a(\lambda) a_{k}(\lambda)\left[\frac{1}{a(\lambda)}-\frac{1}{a_{k}(\lambda)}\right] \sim a(\lambda)^{2}\left(\frac{1}{a(\lambda)}-\frac{1}{a_{k}(\lambda)}\right),
$$

and

$$
\frac{1}{a(\lambda)}-\frac{1}{a_{k}(\lambda)}=L^{\prime}(\rho(\lambda))-L_{k}^{\prime}(\rho(\lambda))+L_{k}^{\prime}(\rho(\lambda))-L_{k}^{\prime}\left(\rho_{k}(\lambda)\right),
$$

where

$$
\begin{aligned}
L^{\prime}(\rho(\lambda))-L_{k}^{\prime}(\rho(\lambda)) & =\sum_{i=k+1}^{\infty} \frac{\log p_{i}}{p_{i}^{\rho(\lambda)}-1} \sim\left(\frac{1}{\rho(\lambda)-1}\right) \frac{1}{(k \log k)^{\rho(\lambda)-1}}, \\
L_{k}^{\prime}(\rho(\lambda))-L_{k}^{\prime}\left(\rho_{k}(\lambda)\right) & =\left(\rho(\lambda)-\rho_{k}(\lambda)\right) L^{\prime \prime}(\rho(\lambda))
\end{aligned}
$$


Using Theorem 1,3), we obtain

$$
L_{k}^{\prime}(\rho(\lambda))-L_{k}^{\prime}\left(\rho_{k}(\lambda)\right) \sim \frac{\lambda L^{\prime \prime}(\rho(\lambda))}{\left|\zeta^{\prime}(\rho(\lambda))\right|(\rho(\lambda)-1) k^{\rho(\lambda)-1}(\log k)^{\rho(\lambda)}} .
$$

Therefore, we have

$$
\frac{1}{a(\lambda)}-\frac{1}{a_{k}(\lambda)} \sim \frac{1}{(\rho(\lambda)-1)(k \log k)^{\rho(\lambda)-1}}
$$

and

$$
a_{k}(\lambda)-a(\lambda) \sim \frac{a^{2}(\lambda)}{\rho(\lambda)-1} \cdot \frac{1}{(k \log k)^{\rho(\lambda)-1}} .
$$

From relations $(2.3),(3.23)$, and $(3.30)$, there exists a constant $\gamma(\lambda)$ depending on $\lambda$ such that

$$
\left|\frac{a_{k}(\lambda)}{p^{\rho_{k}(\lambda)}-1}-\frac{a(\lambda)}{p^{\rho(\lambda)}-1}\right| \leqslant \gamma(\lambda) \frac{\log p}{(\rho(\lambda)-1)(k \log k)^{\rho_{k}(\lambda)-1}} .
$$

Therefore, the proof of Theorem 1 is completed.

\section{Proof of Theorem 2}

We consider the function

$$
t \mapsto H(\underline{x}, t)=\sum_{j=1}^{\omega} \log \left(1+\frac{x_{j}}{t}\right)
$$

We have

$$
H(\underline{x}, t) \leqslant \sum_{j=1}^{\omega} \log \frac{x_{j}}{t}, \quad \text { for any } x_{j}>0 .
$$

The function $t \mapsto H(\underline{x}, t)$ is decreasing from $\infty$ to 0 , when $t \in[0, \infty)$. Thus, there exists a unique $C(\underline{x})>0$ such that

$$
H(\underline{x}, C(\underline{x}))=\log \lambda, \quad \lambda>1 .
$$

Then, for $t=\frac{\Omega(\underline{x})}{\lambda-1}$, we obtain

$$
H(\underline{x}, t)=\log \left(\prod_{j=1}^{\omega}\left(1+(\lambda-1) \frac{x_{j}}{\Omega(\underline{x})}\right)\right) \geqslant \log \left(1+(\lambda-1) \sum_{j=1}^{\omega} \frac{x_{j}}{\Omega(\underline{x})}\right)=\log \lambda .
$$

This implies that

$$
C(\underline{x}) \geqslant \frac{\Omega(\underline{x})}{\lambda-1}
$$


For the upper bound, we write

$$
\log \lambda=\sum_{j=1}^{\omega} \log \left(1+\frac{x_{j}}{C(\underline{x}))}\right) \leqslant \sum_{j=1}^{\omega} \frac{x_{j}}{C(\underline{x})}=\frac{\Omega(\underline{x})}{C(\underline{x})} .
$$

Therefore, we get

$$
\frac{\Omega(\underline{x})}{\lambda-1} \leqslant C(\underline{x}) \leqslant \frac{\Omega(\underline{x})}{\log \lambda} .
$$

Now, we will prove relation (2.11). From equation (2.10), we obtain

$$
T(\underline{x})=\sum_{j=1}^{\omega} \frac{x_{j}}{x_{j}+C(\underline{x})} \leqslant \sum_{j=1}^{\omega} \frac{x_{j}}{C(\underline{x})}=\frac{\Omega(\underline{x})}{C(\underline{x})} \leqslant \lambda-1 .
$$

We set $\gamma_{j}=\frac{x_{j}}{x_{j}+C(\underline{x})}$. One can see that $0 \leqslant \gamma_{j}<1$ and

$$
T(\underline{x})=\sum_{j=1}^{\omega} \gamma_{j} \geqslant \prod_{j=1}^{\omega}\left(1-\gamma_{j}\right)=1-\prod_{j=1}^{\omega} \frac{x_{j}}{x_{j}+C(\underline{x})}=1-\frac{1}{\lambda}=\frac{\lambda-1}{\lambda} .
$$

Hence, we have

$$
\frac{\lambda-1}{\lambda} \leqslant T(\underline{x}) \leqslant \lambda-1 .
$$

Relation (2.12) is immediate from (2.11).

To finish the proof of Theorem 2, first we will use formula (2.9). We take $F=\frac{1}{4}$. So there exist positive constants $C_{1}$ and $C_{2}$ such that

$$
C_{1} \sqrt{\pi} A(\underline{\alpha}) B(\underline{\alpha}) \leqslant H(n, \lambda) \leqslant C_{2} \sqrt{\pi} A(\underline{\alpha}) B(\underline{\alpha}) \text {. }
$$

Second, from the expression of $A(\underline{\alpha})$, we have

$$
C_{1} \sqrt{\pi} \sqrt{\frac{2}{\lambda-1} C(\underline{\alpha})} \frac{\lambda-1}{\lambda \sqrt{\lambda}} \exp (F(\underline{\alpha})) \leqslant H(n, \lambda)
$$

and

$$
H(n, \lambda) \leqslant C_{2} \sqrt{\pi} \sqrt{\frac{2 \lambda}{\lambda-1} C(\underline{\alpha})} \frac{\lambda-1}{\lambda \sqrt{\lambda}} \exp (F(\underline{\alpha})) \frac{1}{(2 \pi)^{k / 2} \sqrt{\alpha_{1} \dot{\alpha}_{2} \cdots \alpha_{k}}} .
$$

By the Stirling's formula $\Gamma(x+1)=x^{x} e^{-x} s(x)$, where $\sqrt{2 \pi x} \leqslant s(x) \leqslant e \sqrt{x}$, $x \geqslant 1$, the relation $F(\underline{\alpha}) \leqslant \rho_{k}(\lambda) \log n$ and equations (2.10), (2.11) imply equation (2.13). Notice that the inequality $F(\underline{\alpha}) \leqslant \rho_{k}(\lambda) \log n$ can be obtained by the use of the result given in [13, Lemma 3.5], with $A=\log n, \rho_{k}=\rho_{k}(\lambda), a_{k}=a_{k}(\lambda)$. This completes the proof of Theorem 2 . 


\section{Proof of Theorem 3}

We start by proving inequality (2.15). Put

$$
\theta_{1}(\lambda)=\frac{\lambda}{(\rho(\lambda)-1)\left|\zeta^{\prime}(\rho(\lambda))\right|}
$$

From Theorem 2 and inequality (2.13), we have

$$
\log H(n, \lambda) \leqslant \log \left(\frac{C_{2}}{\lambda} \sqrt{\frac{\lambda-1}{\log \lambda}}\right)-\frac{k}{2} \log \pi+\rho_{k}(\lambda) \log n .
$$

Write

$$
\rho_{k}(\lambda) \log n=\rho(\lambda) \log n-\left(\rho(\lambda)-\rho_{k}(\lambda)\right) \log n .
$$

By Theorem 1, there exists a positive constant $\theta_{1}(\lambda)$ such that

$$
\rho(\lambda)-\rho_{k}(\lambda) \geqslant \frac{\theta_{1}(\lambda)}{k^{\rho(\lambda)-1}(\log k)^{\rho(\lambda)}} .
$$

For $n \geqslant 16$, we have $\log \log n>1$. Thus, for $2 \leqslant k \leqslant \frac{(\log n)^{1 / \rho(\lambda)}}{\log \log n}<\log n$, we obtain

$$
\rho(\lambda)-\rho_{k}(\lambda) \geqslant \frac{\theta_{1}(\lambda)}{k^{\rho(\lambda)-1}(\log k)^{\rho(\lambda)}} \geqslant \theta_{1}(\lambda) \frac{(\log n)^{\frac{1}{\rho(\lambda)}-1}}{\log \log n}
$$

and for $k>\frac{(\log n)^{1 / \rho(\lambda)}}{\log \log n}$, we get

$$
\frac{k}{2} \log \pi>\frac{1}{2} \frac{(\log n)^{1 / \rho(\lambda)}}{\log \log n} .
$$

Therefore, we deduce that

$$
\begin{aligned}
\left(\rho(\lambda)-\rho_{k}(\lambda)\right) \log n+\frac{k}{2} \log \pi & \geqslant \min \left(\frac{1}{2}, \theta_{1}(\lambda)\right) \frac{(\log n)^{1 / \rho(\lambda)}}{\log \log n} \\
& =\gamma_{1}(\lambda) \frac{(\log n)^{1 / \rho(\lambda)}}{\log \log n} .
\end{aligned}
$$

For a large positive integer $n$, we deduce inequality (2.15).

Now, we will prove inequalities (2.16). For this, we take a large $H$-champion number $N$. We apply [13, Lemme 3.5], with $A=\log n, k=\left[\alpha \frac{(\log n)^{1 / \rho(\lambda)}}{\log \log n}\right]$, and $0<\alpha<\rho(\lambda) a(\lambda)^{1 / \rho(\lambda)}$. So, for a large $H$-champion number $n=2^{\alpha_{1}} \cdot 3^{\alpha_{2}} \cdots p_{k}^{\alpha_{k}}$, we follow the procedure used in [13] (see pages 1694-1697) to obtain formula (2.16).

By Theorem 2 and inequality (2.13), we have

$$
\log H(n, \lambda) \geqslant F(\underline{\alpha})-k-\frac{1}{2} \sum_{i=1}^{k} \log \alpha_{i}+\log g(\lambda) .
$$


On the other hand, we know that

$$
F(\underline{\alpha}) \geqslant F\left(\underline{x^{\star}}\right)-\rho(\lambda) \log 2-k,
$$

where $\underline{x^{\star}}$ satisfies

$$
F\left(\underline{x^{\star}}\right)=\rho(\lambda) \log n,
$$

see [13, Lemme 3.5]. Therefore, we get

$\log H(n, \lambda) \geqslant \rho(\lambda) \log n-\left(\rho(\lambda)-\rho_{k}(\lambda)\right) \log n-2 k-\frac{1}{2} \sum_{i=1}^{k} \log \alpha_{i}+\log g(\lambda)-\rho(\lambda) \log 2$.

Using equation (2.3) of Theorem 1, we obtain

$$
\left(\rho(\lambda)-\rho_{k}(\lambda)\right) \log n=O\left((\log n)^{\frac{1}{\rho(\lambda)}} / \log \log n\right)=O(k)
$$

and

$$
\frac{1}{2} \sum_{i=1}^{k} \log \alpha_{i}=O(k)
$$

See [13, Lemme 4.2]. Hence, we deduce that

$$
\log H(n, \lambda) \geqslant \rho(\lambda) \log n-\gamma_{2}(\lambda) \frac{(\log n)^{\frac{1}{\rho(\lambda)}}}{\log \log n}+\log g(\lambda)-\rho(\lambda) \log 2,
$$

for some positive constant $\gamma_{2}$. Therefore, we complete the proof of the second part of Theorem 3.

It remains to prove the third part of Theorem 3. So let $k=\omega(N)$. From equation (2.13), we have

$$
\log H(N, \lambda) \leqslant \rho_{k}(\lambda) \log N-\frac{k}{2} \log \pi+\log f(\lambda)
$$

and

$$
\log H(N, \lambda) \leqslant \rho(\lambda) \log N-\left(\rho\left(\lambda-\rho_{k}(\lambda)\right) \log N-\frac{k \log \pi}{2}+\log f(\lambda) .\right.
$$

Thus, we get

$$
\left(\rho\left(\lambda-\rho_{k}(\lambda)\right) \log N \leqslant \rho(\lambda) \log N-\log H(N, \lambda)-k \frac{\log \pi}{2}+\log f(\lambda) .\right.
$$

From Theorem 3, 1), we obtain

$$
\left(\rho(\lambda)-\rho_{k}(\lambda)\right) \log N \leqslant \gamma_{1}(\lambda) \frac{(\log N)^{1 / \rho_{\lambda}}}{\log \log N}+\log \left(\frac{f(\lambda)}{g(\lambda)}\right) .
$$

One can see that there exists $C>0$ such that

$$
k^{\rho(\lambda)-1}(\log k)^{\rho(\lambda)} \geqslant C(\log N)^{1-1 / \rho(\lambda)} \log \log N .
$$


Note that the function $g: t \mapsto t^{\rho(\lambda)-1}(\log t)^{\rho(\lambda)}$ tends to infinity when $t \rightarrow \infty$ and is increasing, for $t \geqslant 1$. Its inverse function satisfies $g^{-1}(y) \sim\left(\frac{y}{\log (y)^{\rho}}\right)^{\frac{1}{\rho-1}}$ as $y \rightarrow \infty$. Then, we get

$$
k \geqslant g^{-1}\left(C(\lambda) \frac{(\log N)^{1-1 / \rho(\lambda)}}{\log \log N}\right) \sim C^{\prime}(\lambda) \frac{(\log N)^{1 / \rho(\lambda)}}{\log \log N}, \quad C^{\prime}(\lambda)>0 .
$$

Therefore, we obtain the lower bound for $k=\omega(N)$

$$
\omega(N)=k \geqslant \gamma_{3}(\lambda) \frac{(\log N)^{1 / \rho_{\lambda}}}{\log \log N} .
$$

Similarly, we obtain the second inequality. This completes the proof of Theorem 3.

\section{Proof of Theorem 4}

Let $N=2^{\alpha_{1}} \cdot 3^{\alpha_{2}} \cdots p_{k}^{\alpha_{k}}, \alpha_{1}, \alpha_{2}, \ldots \alpha_{k} \geqslant 1$, where $p_{k}$ is the $k^{\text {th }}$ prime number. We have $\log N=\sum_{i=1}^{k} \alpha_{i} \log p_{i}$. We consider the maximization problem for the function

$$
F(\underline{x})=\sum_{j=1}^{k} x_{j} \log \left(1+\frac{C(\underline{x})}{x_{j}}\right), \quad \underline{x}=\left(x_{1}, x_{2}, \ldots, x_{k}\right)
$$

on the set

$$
\mathcal{D}_{N}=\left\{\underline{x} \in \mathbb{R}_{+}^{* k}: \sum_{j=1}^{k} x_{j} \log p_{j} \leqslant \log N\right\} .
$$

This problem is equivalent to the following one

$$
\left\{\begin{array}{l}
\sum_{j=1}^{k} x_{j} \log p_{j}=\log N \\
\max F(\underline{x}) .
\end{array}\right.
$$

Referring to [9, Lemma 6] and [13], there exists a unique $\underline{x}^{*}=\left(x_{1}^{*}, x_{2}^{*}, \ldots, x_{k}^{*}\right) \in$ $\mathcal{D}_{N}$ such that

$$
\left.\log N=\sum_{i=1}^{k} x_{i} \log p_{i}\right), \quad C\left(\underline{x}^{*}\right)=a_{k}(\lambda) \log N
$$

and

$$
x_{i}^{*}=\frac{a_{k}(\lambda) \log N}{p_{i}^{\rho_{k}(\lambda)}-1}, \quad F\left(\underline{x}^{*}\right)=\rho_{k}(\lambda) \log N .
$$

Moreover, for $\underline{\beta}=\left(\beta_{1}, \beta_{2}, \ldots, \beta_{k}\right) \in \mathcal{D}_{N}$, we have

$$
F\left(\underline{x}^{*}\right) \geqslant F(\underline{\beta})+\frac{1}{4 \log N \log p_{k}} \sum_{i=1}^{k}\left(\beta_{i}-x_{i}^{*}\right)^{2}\left(\log p_{i}\right)^{2}, \quad \forall k \geqslant 2 .
$$


If $N$ is a $H$-champion number, then we have

$$
\log p_{k} \sim \frac{1}{\rho(\lambda)} \log N \log N, \quad \text { and } \quad \sum_{i=1}^{k}\left(\beta_{i}-x_{i}^{*}\right)\left(\log p_{i}\right)=O(\log N)^{\delta(\lambda)}
$$

Now, we can start the proof of Theorem 4 . We set

$$
\beta_{i}=\frac{a(\lambda)}{p_{i}^{\rho(\lambda)}-1} \quad \text { and } \quad k=\omega(N) .
$$

Then, we have

$$
\left|\sum_{i=1}^{k}\left(\alpha_{i}-\beta_{i} \log N\right)\right| \leqslant \sum_{i=1}^{k}\left|\alpha_{i}-\beta_{i} \log N\right| \leqslant \sum_{i=1}^{k}\left|\alpha_{i}-x_{i}^{*}\right|+\sum_{i=1}^{k}\left|x_{i}^{*}-\beta_{i} \log N\right|,
$$

where

$$
\sum_{i=1}^{k}\left|\alpha_{i}-x_{i}^{*}\right| \leqslant O(\log N)^{\delta(\lambda)}
$$

and

$$
\begin{aligned}
\sum_{i=1}^{k}\left|x_{i}^{*}-\beta_{i} \log N\right| & =(\log N) \sum_{i=1}^{k}\left|\frac{a_{k}(\lambda)}{p_{i}^{\rho_{k}(\lambda)}-1}-\frac{a(\lambda)}{p_{i}^{\rho(\lambda)}-1}\right| \\
& \leqslant \frac{\gamma(\lambda)(\log N)}{(k \log k)^{\rho(\lambda)-1}} \sum_{i=1}^{k} \frac{\log p_{i}}{p_{i}^{\rho(\lambda)}}=O\left(\frac{\log N}{k^{\rho(\lambda)-1}}\right) .
\end{aligned}
$$

By Theorem 3. 2), we have

$$
k \geqslant \gamma_{3}(\lambda) \frac{(\log N)^{1 / \rho(\lambda)}}{\log N \log N} .
$$

Thus, we get

$$
\frac{\log N}{k^{\rho(\lambda)-1}}=O\left((\log N)^{1 / \rho(\lambda)}(\log \log N)^{\rho(\lambda)-1}\right)=O\left((\log N)^{\delta(\lambda)}\right),
$$

where $\rho(\lambda)>1$. One can see that

$$
\sum_{i=k+1}^{\infty} \beta_{i}=\sum_{i=k+1}^{\infty} \frac{a(\lambda)}{p_{i}^{\rho(\lambda)}-1} \leqslant 2 a(\lambda) \sum_{i=k+1}^{\infty} \frac{1}{p_{i}^{\rho(\lambda)}} \leqslant \frac{2 a(\lambda)}{(\rho(\lambda)-1) k^{\rho(\lambda)-1}} .
$$

Hence, we have

$$
\left|\Omega(N)-\sum_{i=1}^{\infty} \beta_{i} \log N\right| \leqslant O(\log N)^{\delta(\lambda)} .
$$


For $1 \leqslant i \leqslant k$, we obtain

$$
\left|\alpha_{i}-x_{i}^{*}\right| \ll \frac{(\log N)^{\delta(\lambda)}}{p_{i}}
$$

and

$$
\left|x_{i}^{*}-\beta_{i} \log N\right| \leqslant \gamma(N) \frac{\log p_{i}}{p_{i}^{\rho(\lambda)}} \frac{\log N}{(k \log k)^{\rho(\lambda)-1}}=\frac{1}{\log p_{i}} O\left(\frac{\log N}{k^{\rho(\lambda)-1}}\right), \quad \forall i .
$$

We deduce that

$$
\left|x_{i}^{*}-\beta_{i} \log N\right| \ll \frac{(\log N)^{1 / \delta(\lambda)}}{\log p_{i}}
$$

and

$$
\left|x_{i}^{*}-\beta_{i} \log N\right| \leqslant O\left(\frac{(\log N)^{\delta(\lambda)}}{\log p_{i}}\right), \quad \forall 1 \leqslant i \leqslant k .
$$

This shows the first part of Theorem 4 .

One can see that

$$
\begin{aligned}
& |C(\underline{\alpha})-C(\underline{\beta}) \log N| \leqslant \sum_{i=1}^{k}\left|\alpha_{i}-\beta_{i} \log N\right|+\sum_{i=1}^{\infty} \beta_{i} \log N=O(\log N)^{\delta(\lambda)}, \\
& \lambda=\zeta(\rho(\lambda))=\prod_{i=1}^{\infty}\left(1-p_{i}^{-\rho(\lambda)}\right)^{-1}=\prod_{i=1}^{\infty} \frac{p_{i}^{\rho(\lambda)}}{p_{i}^{\rho(\lambda)}-1}=\prod_{i=1}^{\infty}\left(1+\frac{1}{p_{i}^{\rho(\lambda)}-1}\right) .
\end{aligned}
$$

Therefore, equations

$$
C\left(\frac{1}{2^{\rho(\lambda)}-1}, \ldots\right)=1 \quad \text { and } \quad C\left(\frac{a(\lambda)}{2^{\rho(\lambda)}-1}, \frac{a(\lambda)}{3^{\rho(\lambda)}-1} \ldots\right)=a(\lambda)
$$

imply that

$$
C(\underline{\beta})=a(\lambda)
$$

Thus, the second part of Theorem 4 is proved.

From Theorem 4 1) and relation (2.6), we obtain

$$
T(\underline{\alpha})=\zeta_{\mathcal{P}}(\rho(\lambda))+O\left((\log N)^{\delta(\lambda)-1}\right) .
$$

Using Theorem 4. 2), 3) and equation (2.8), we complete the proof of Theorem 4.

\section{Comments on numerical computations}

In this section, we do some numerical computations to study the behavior of some constants. Let $\lambda$ be a complex number, $k$ a positive integer, and $\mathcal{P}_{k}$ the set of 
primes numbers defined by $\mathcal{P}_{k}=\left\{p_{1}, p_{2}, \ldots, p_{k}\right\}$, with $p_{1}=2<p_{2}=3<\cdots<p_{k}$. We know that

$$
\zeta_{k}(s)=\prod_{p \in \mathcal{P}_{k}}\left(1-p^{-s}\right)^{-1},
$$

(see $(2.1)), \rho_{k}(\lambda)$ is a positive real solution to $\zeta_{k}(s)=\lambda$ and

$$
a_{k}(\lambda)=-\frac{\lambda}{\zeta_{k}^{\prime}\left(\rho_{k}(\lambda)\right)}
$$

Moreover, we consider

$$
p_{k}(\lambda)=\frac{\lambda}{\left(\rho_{k}(\lambda)-1\right) \zeta_{k}^{\prime}\left(\rho_{k}(\lambda)\right)}
$$

the truncated part of the coefficient in equation (2.3) and

$$
\delta(\lambda)=\frac{1}{2}\left(1+\frac{1}{\rho(\lambda)}\right),
$$

(see Theorem 4). We wrote a program in Maple that we run under a MacBook Air. We restricted the computations to $3 \leqslant \lambda \leqslant 7$ and $3 \leqslant k \leqslant 20$, because the result is known for $\lambda=2$ and the computations become extremely slow for higher values of $\lambda$ and $k$. Here are some comments on these computations, which confirm the properties that we proved:

1. As we consider $\rho_{k}(\lambda)>1$, we notice that the first value of $\rho_{k}(\lambda)$ is obtained for higher values of $k$ when $\lambda$ is higher.

2. The values of $\rho_{k}(\lambda)$ increase when $k$ increases. They are smaller when $\lambda$ increases. But in all cases, we have $\rho_{k}(\lambda)<2$.

3 . When $k$ increases, the values of $a_{k}(\lambda), p_{k}(\lambda), p_{k}(\lambda), \delta_{k}\left(\rho_{k}\right)$ decrease. We notice that as $\lambda$ increases, the behavior is different. In all cases, we see that

$$
a_{k}(\lambda)<0.772, \quad p_{k}(\lambda)<125.406, \quad \delta_{k}\left(\rho_{k}\right)<1 .
$$

Acknowledgements. The work on this paper started during a visit of the first author at Purdue University North Central (USA) and continued during the visits of the second and third authors at Université d'Evry Val d'Essonne (France). They thank these institutions for the fruitful atmosphere of collaboration.

\section{References}

[1] A. Bayad, Arithmetical properties of elliptic Bernoulli and Euler numbers, Int. Journal of Algebra, Vol. 4 (2010), 8, 353-372.

[2] L. Carlitz, V.E. Hoggart, Generalized Eulerian numbers and polynomials, Math. Magazine 32 (1959), 247-260.

[3] L. Carlitz, Extended Bernoulli and Eulerian numbers, Duke Mathematical Journal 31 (1964), 4, 667-689.

[4] E.R. Canfield, P. Erdôs, C. Pomerance, On a Problem of Oppenheim concerning "Factorisatio Numerorum", J. Number Theory 17 (1983), 1-28. 
[5] B. Chor, P. Lemke and Z. Mador, On the number of ordered factorizations of natural numbers, Discrete Math. 214 (2000), 123-133.

[6] D. Coppermith and M. Lewenstein, Construction bounds on ordered factorizations, SIAM J. Discrete Math. 19 (2005), 301-303.

[7] P. Dussart, The $k^{\text {th }}$ prime is greater than $k(\log k+\log \log k-1)$ for $k \geqslant 2$, Math. Comp. 68 (1999), 411-415.

[8] P. Erdôs, On some asymptotic formulas in the theory of the "factorisatio numerorum", Ann. of Math. 42 (1941), 989-993. Corrections to two of my papers, Ann. of Math. 44 (1943), 647-651.

[9] R. Evans, An asymptotic formula for extended Eulerian numbers, Duke Math. J. 41 (1974), 161-175.

[10] E. Grosswald, Verallgemeinerte Eulersche Zahlen, Math. Z. 140 (1974), 173177.

[11] G.H. Hardy and S. Ramanujan, Asymptotic formulce for the distribution of integers of various types, Proc. London Math. Soc. 16 (1917), 112-132 and Collected Papers of S. Ramanujan, Cambridge University Press, 245-261.

[12] G.H. Hardy and S. Ramanujan, Asymptotic formulce in combinatory analysis, Proc. London Math. Soc. 2 (1918), 75-115 and Collected Papers of S. Ramanujan, Cambridge University Press, 276-309.

[13] M. Deleglise, M.O. Hernane, J.-L. Nicolas, Grandes valeurs et nombres champions de la fonction arithmétique de Kalmár, J. Number Theory 128 (2008), $1676-1716$.

[14] E. Hille, A Problem in "Factorisatio Numerorum", Acta Arith. 2 (1936), 134144.

[15] H.-K. Hwang, Distribution of the number of factors in random ordered factorizations of integers, J. Number Theory 81 (2000), 61-92.

[16] S. Ikehara, On Kalmár's problem in "Factorisation Numerorum", Proc. Phys.Math. Soc. Japan 21 (1939), 208-219; II, 23 (1941), 767-774.

[17] L. Kalmár, A "Factorisatio Numerorum" problémàjàròl, Mathematikai ès Fisikai Lapok 38 (1931), 1-15.

[18] M. Klazar and F. Luca, On the maximal order of numbers in the "factorizatio numerorum" problem, J. Number Theory 124 (2007), 470-490.

[19] D.-H. Lehmer, Generalized Eulerian numbers, J. of Combinatorial Theory, series A, 32 (1982), 195-215.

[20] P.A. MacMahon, Dirichlet's series and the theory of partitions, Proc. London Math. Soc. 22 (1924), 404-411.

[21] N.P. Nörlund, Vorlesungen über Differenrechnungen, Berlin, 1924.

Addresses: Abdelmejid Bayad: Département de mathématiques, Université d'Evry Val d'Essonne, 23 Bd. De France, 91037 Evry Cedex, France;

Mohand Ouamar Hernane: Université des Sciences et de la Technologie, Faculté de Mathématiques, BP 32, USTHB, 16123 Bab-Ezzouar Alger, Algérie;

Alain Togbé: Mathematics Department, Purdue University North Central, 1401 S, U.S. 421, Westville IN 46391, USA.

E-mail: abayad@maths.univ-evry.fr, mhernane@usthb.dz, atogbe@pnw.edu

Received: 28 September 2015; revised: 16 February 2016 